\title{
UMA PROPOSTA DE ENSINO DA TRANSFERÊNCIA DE CALOR EM ALETAS EM REGIME ESTACIONÁRIO E CONVECÇÃO NATURAL
}

RenatoLetizia Garcia-renato-garcia@uergs.edu.br

Universidade Estadual do Rio Grande do Sul - Unidade de Porto Alegre

Av. Bento Gonçalves, 8855 - bairro Agronomia

CEP 91450-000 - Porto Alegre - RS

Marco CésarSauer-sauer@liberato.com.br

Fundação Escola Técnica Liberato Salzano Vieira da Cunha-Curso de Eletrônica

Rua Incofidentes, 395 - bairro Primavera

CEP 93340-140 - Novo Hamburgo - RS

Edson Abel dos Santos Chiaramonte-edsonchiaramonte@unipampa.edu.br

Universidade Federal do Pampa - Campus Bagé

Av. Maria Anunciação Gomes de Godoy, 1650 - bairro Malafaia

CEP 96413-172 - Bagé - RS

Luciano Andreatta Carvalho da Costa - luciano-costa@uergs.edu.br Universidade Estadual do Rio Grande do Sul - Unidade de Porto Alegre

Av. Bento Gonçalves, 8855 - bairro Agronomia

CEP 91450-000 - Porto Alegre - RS

Michele Espinosada Cunha-michele-cunha@uergs.edu.br

Universidade Estadual do Rio Grande do Sul - Unidade de Porto Alegre

Av. Bento Gonçalves, 8855 - bairro Agronomia

CEP 91450-000 - Porto Alegre - RS

Resumo: O trabalho descreve um experimento didático sobre dissipação de calor, em regime estacionário, em aletas circulares aquecidas na base e expostas ao ar ambiente, propondo uma modificação na metodologia tradicionalmente utilizada na estimativa do coeficiente convectivo de transferência de calor. Os resultados obtidos são comparados com valores teóricos previstos em correlações empíricas que relacionam esse coeficiente com números adimensionais, comprovando a adequação da proposição formulada. A estratégia de ensino utilizada na formulação dessa proposta e na análise dos resultados é confrontada com o modelo de ensino tradicional, destacando as vantagens advindas da realização de uma aula prática como forma de minimizar as dificuldades enfrentadas pelos alunos no estudo da transferência de calor em aletas.

Palavras-chave: Aletas. Transferência de calor. Convecção natural. Radiação do calor. 


\section{INTRODUÇÃO}

A estratégia de utilizar aletas como forma de dissipação de calor é largamente empregada em equipamentos industriais, eletrodomésticos e outros objetos de uso cotidiano. Consequentemente, torna-se necessário quantificar o calor transferido através de um arranjo de aletas, que atua como extensão da superfície de troca térmica de um corpo sólido.

Nesse contexto, disciplinas de graduação que tratam da transferência de calor e massa incluem um tópico destinado ao estudo da transferência de calor em aletas. Tais disciplinas, presentes na grade curricular de engenharia química e mecânica, e cursos afins, tradicionalmente abordam esse tópico a partir de um balanço térmico diferencial que possibilita calcular o perfil de temperatura e o calor dissipado em uma aleta individual (LIENHARD, 2004).

A abordagem descrita no parágrafo acima é fundamentada nos mecanismos de condução e convecção do calor e demonstra a importância do estudo das equações diferenciais e sua aplicação em problemas de engenharia. Contudo, o modelo convencional de ensino aplicado nas disciplinas que tratam do assunto, baseado em aulas expositivas e resolução de problemas teóricos, nem sempre capacita o aluno aplicar os conhecimentos adquiridos em situações físicas reais. A realização de aulas práticas, com um estudo prévio dos fenômenos físicos observados, e uma discussão criteriosa dos resultados obtidos, é uma forma de capacitar o aluno na análise dessas situações (ARAÚJO; ABIB, 2003).

Algumas instituições de ensino superior possuem laboratórios com módulos didáticos comerciais, ou desenvolveram equipamentos específicos, que permitem o estudo experimental da transferência de calor (GARCIA et al., 2017). No que diz respeito ao estudo da transferência de calor em superfícies estendidas (aletas), um dos equipamentos mais utilizados consiste de um conjunto constituído de uma ou mais aletas longas, engastadas em uma caixa que promove o aquecimento e mantém a temperatura constante na base da aleta, expondo sua superfície ao ar ambiente. Este equipamento é dotado de sensores que registram a temperatura ao longo da aleta, possibilitando que o aluno obtenha o perfil de temperatura e realize cálculos a partir dos conhecimentos teóricos previamente adquiridos. O ensaio experimental é conduzido sem circulação forçada de ar, de modo que a movimentação de ar ocorre como consequência da convecção natural induzida pelo aquecimento da aleta.

Nesse trabalho, utilizando dados experimentais do perfil temperaturas, será avaliada a metodologia comumente empregada no estudo da transferência de calor em aletas. Os autores irão demonstrar a importância do mecanismo de radiação no processo de dissipação do calor em uma aleta. Nesse intuito, serão descritos inicialmente os fundamentos teóricos desse assunto e, na sequencia, propostas algumas correções que possibilitam considerar, simultaneamente, a radiação e a convecção de calor na aleta. $\mathrm{Na}$ seção 3 , são relatados os ensaios realizados e os resultados obtidos. $\mathrm{Na}$ discussão subsequente, são comparados os dados obtidos segundo o modelo tradicional e a metodologia proposta nesse artigo, avaliando eventuais fontes de erros. Finalizando o artigo, são feitas algumas considerações sobre melhorias no processo de aprendizagem da transferência de calor em aletas advindas da proposta de ensino apresentada.

\section{FUNDAMENTOS TEÓRICOS}

O aumento da taxa de transferência de calor a partir da inserção de aletas em uma placa plana, conforme ilustrado na Figura 1, é avaliado através de um balanço térmico aplicado em um elemento infinitesimal de volume da aleta. 
Figura 1 - Aumento da transferência de calor com a inserção de aletas numa superfície.

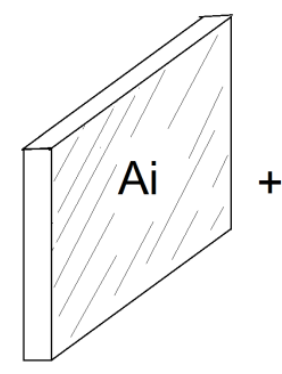

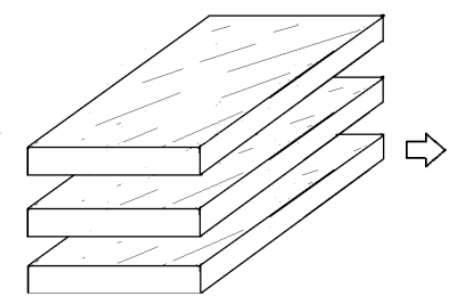

n Aletas

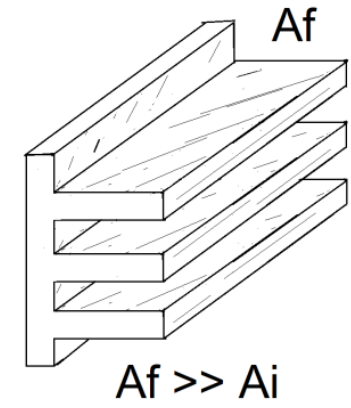

Af $>>\mathrm{Ai}$

O balanço térmico entre duas seções transversais, afastadas por uma distância $\mathrm{dx}$, ilustrado na Figura 2 para uma aleta circular, resulta na seguinte equação diferencial:

$$
\frac{d^{2} T}{d x^{2}}-m^{2} \cdot\left(T-T_{a m b}\right)=0
$$

na qual $\mathbf{T}$ é a temperatura numa seção transversal e $\mathbf{T}_{\mathbf{a m b}}$ é a temperatura ambiente. Nesta equação, m é um termo que relaciona a condutividade térmica do material da aleta, o coeficiente convectivo de transferência de calor, o perímetro e a área da seção tranversal de uma aleta, respectivamente, $\mathbf{k}, \mathbf{h}, \mathbf{P}$ e $\mathbf{A}$, através da expressão:

$$
m=\sqrt{\frac{h \cdot P}{k \cdot \mathrm{A}}}
$$

Figura 2 - Balanço térmico em uma aleta circular.

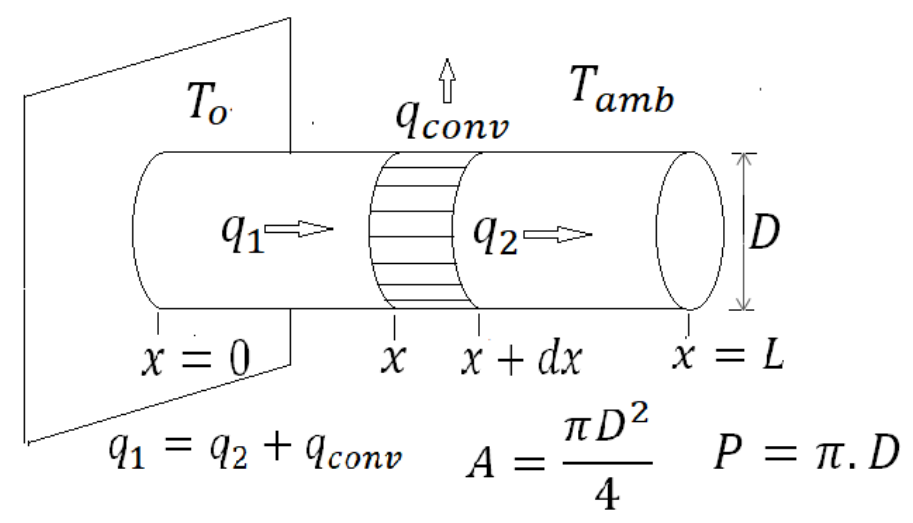

A modelagem utlizada na obtenção da Equação (2) considera o fluxo de calor unidimensional, em regime permanente, numa aleta de seção transversal constante. O calor flui por condução no interior da aleta, sendo transferido por convecção na interface sólida com o ar ambiente, conforme ilustrado na Figura 2. A solução geral da Equação (2), que descreve o perfil de temperatura ao longo da aleta, é:

$$
T(x)=C_{1} \cdot e^{m \cdot x}+C_{2} \cdot e^{-m \cdot x}
$$


na qual $\mathrm{C}_{1}$ e $\mathrm{C}_{2}$ são constantes arbitrárias a serem determinadas pelas condições de contorno na base e na extremidade exposta da aleta. A situação mais simples, válida para aletas de "comprimento infinito", resulta em:

$$
T(x)=T_{a m b}+\left(T_{0}-T_{a m b}\right) \cdot e^{-m \cdot x}
$$

na qual $\mathrm{T}_{0}$ é a temperatura na base da aleta, e a temperatura na outra extremidade da aleta é arbitrada como sendo igual à temperatura ambiente. Uma aleta de "comprimento infinito" é aquela cuja temperatura da ponta (extremidade oposta à base), pode ser igualada à temperatura ambiente, sendo tal condição associada ao seguinte critério:

$$
\text { m. } L \geq 2,51
$$

\subsection{O coeficiente convectivo de transferência de calor}

A metodologia de ensino amplamente empregada em aulas expositivas sobre transferência de calor consiste em explanações do conteúdo teórico, que inclui a análise e/ou dedução de equações, seguida da resolução de problemas. A determinação do perfil de temperaturas em um ponto da aleta afastado a uma determinada distância da base é um dos problemas usualmente propostos, para o qual são fornecidos dados relativos à geometria e condutividade térmica do material constituinte da aleta, além dos valores da temperatura ambiente e na base da aleta. O coeficiente convectivo de transferência de calor, $\mathbf{h}$, é outra informação necessária à resolução desse tipo de problema; embora seu valor seja, essencialmente, uma estimativa para uma situação real, diferentemente dos demais dados fornecidos no enunciado.

$\mathrm{Na}$ abordagem descrita, a obtenção do resultado numérico correto é uma das maiores preocupações do aluno ao efetuar os cálculos, sendo induzido a pensar que as equações utilizadas reproduzem, com elevado grau de confiabilidade, o sistema físico descrito no enunciado. Cabe destacar que o valor de $\mathbf{h}$, na convecção natural de um gás, situa-se entre 2 e $25 \mathrm{~W} / \mathrm{m}^{2} . \mathrm{K}$, segundo a literatura científica (INCROPERA; DE WITT, 2008); o que significa uma diferença relativa da ordem de $1000 \%$ entre os limites desse intervalo. Esse fato, muitas vezes, não é destacado com a devida importância nas aulas expositivas ministradas sobre o assunto; gerando alguma desconexão entre o ensino formal, associado à resolução de problemas teóricos, e o comportamento real do sistema físico descrito nesses problemas.

Atividades experimentais, cuja realização tende a motivar e envolver o aluno no assunto em pauta, aumentando sua capacidade de aprendizado (GIORDAN, 1999), possibilitam confrontar o comportamento real com as simulações teóricas previstas para um sistema físico. Um dos dispositivos mais empregados no estudo experimental da transferência de calor em aletas registra as temperaturas ao longo de uma barra metálica cilíndrica, na posição horizontal. Uma das extremidades da barra é aquecida e mantida à temperatura constante. Após algum tempo, é atingido o regime estacionário, e o perfil de temperaturas correspondente é utilizado para avaliar o parâmetro m. Na maioria dos casos, o dispositivo possui várias barras, de materiais e/ou diâmetros distintos, possibilitando a comparação dos valores obtidos para esse parâmetro. Além disso, é possível calcular o valor de $\mathbf{h}$, e realizar uma comparação similar.

Nesse ponto cabe uma análise a respeito da natureza e das características desse coeficiente, também denominado coeficiente de película, na situação analisada. $O$ aquecimento de um sólido induz a presença de um gradiente de temperatura junto à interface 
que o delimita, de modo que se forma uma película de fluido, de espessura diminuta, responsável pela transferência de calor entre a superfície exposta do objeto e o fluido que o circunda. Este gradiente de temperatura induz a movimentação do fluido na interface, num processo denominado convecção natural. As temperaturas da interface e do fluido circundante, bem como a geometria do sólido e as propriedades termofísicas do fluido contido na película descrita, determinam o valor do coeficiente convectivo de transferência de calor.

\subsection{Números dimensionais utilizados no cálculo do valor teórico de $h$}

No estudo da convecção do calor, utiliza-se comumente a adimensionalização de equações e a combinação de variáveis em números adimensionais (ÇENGEL; GHAJAR, 2012). O coeficiente de película, h, é adimesionalizado na forma do número de Nusselt:

$$
N u=\frac{h \cdot L_{c}}{k_{f}}
$$

sendo $\mathbf{k}_{\mathbf{f}}$ a condutividade térmica do fluido (ar) na temperatura de filme; e $\mathbf{L}_{\mathfrak{c}}$, o comprimento característico da aleta. O termo filme é outra designação para a película de ar em que se forma o gradiente de temperatura entre a aleta e o ambiente, e cuja temperatura é arbitrada como sendo a média entre a superfície da aleta e o ar circundante. O comprimento característico, para uma barra cilíndrica horizontal, corresponde ao seu diâmetro. O número de Grashoff (Gr), associado ao fenômeno da convecção natural, é calculado através da expressão

$$
G r=\frac{g \cdot \beta \cdot \Delta T \cdot L_{C}^{3}}{v^{2}}
$$

sendo $\boldsymbol{g}$ a aceleração da gravidade; $\boldsymbol{\beta}$, o coeficiente de expansão térmica do fluido; $\Delta \mathbf{T}$, a diferença entre a temperatura da aleta e do fluido; e $\mathbf{v}$, a difusividade cinemática. $\mathrm{O}$ coeficiente de expansão térmica corresponde ao inverso da temperatura (absoluta) de filme.

Outros dois números adimensionais envolvidos na convecção natural são o de Prandtl (Pr) e o de Rayleigh $(\mathrm{Ra})$, assim definidos:

$$
\operatorname{Pr}=\frac{v}{\alpha} \quad, \quad R a=G r \cdot \operatorname{Pr}
$$

A movimentação do fluido numa interface sólida, num processo de convecção natural, é influenciada pela geometria dessa interface, havendo uma correlação específica, entre os números adimensionais citados, específica para cada geometria. No caso de um cilindro horizontal longo, para $10^{2}<\mathrm{Ra}<10^{4}$. é válida a relação (MORGAN, 1975):

$$
N u=0,85 \cdot R a^{0,188}
$$

O cálculo do coeficiente de película teórico, associado à convecção natural do ar numa barra cilíndrica longa e horizontal, em regime estacionário, é feito a partir das Equações (6) a (10). No intuito de simplificar os cálculos, a temperatura da aleta foi considerada como sendo o valor médio entre a temperatura da sua base e o ar ambiente. 


\subsection{Radiação e convecção de calor na superfície da aleta}

O valor experimental do parâmetro $\mathbf{m}$ é obtido a partir dos registros de temperatura ao longo da barra cilíndrica, num ensaio real, e do ajuste da função exponencial descrita na Equação (4) a esse conjunto de registros. A Equação (2) fornece o valor de h associado ao parâmetro $\mathrm{m}$ calculado através desse ajuste.

O balanço térmico tradicionalmente adotado em uma aleta, que resulta na equação diferencial indicada em (1), considera que o calor é transferido da superfície metálica para o ar ambiente exclusivamente por convecção. Tal hipótese não condiz com os resultados do ensaio mencionado, pois podem ocorrer discrepâncias significativas entre o valor experimental de $\mathrm{h}$ e o valor teórico desse coeficiente, conforme será visto na seção 3 . Consequentemente, o modelo proposto deve ser reformulado, incorporando-se ao balanço original a parcela de calor transferida por radiação. A taxa de transferência de calor por radiação, $\mathbf{q}_{\text {rad., }}$ entre uma superfície sólida e o ar ambiente pode ser assim expressa:

$$
q_{\text {rad. }}=\varepsilon \cdot \sigma \cdot A_{e} \cdot\left(T^{4}-T_{a m b}^{4}\right)
$$

sendo $\boldsymbol{\varepsilon}, \boldsymbol{\sigma}$ e $\mathbf{A}_{\mathbf{e}}$, respectivamente, a emissividade da superfície sólida, a constante de StefanBoltzmann, cujo valor é $5,67 \times 10^{-8} \mathrm{~W} /\left(\mathrm{m}^{2} \cdot \mathrm{K}^{4}\right)$, e a $\mathrm{A}_{\mathrm{e}}$, a área exposta da superfície. A emissividade é um valor numérico entre 0 e 1 , e os valores de $\mathrm{T}$ e $T_{\mathrm{amb}}$. correspondem às temperaturas absolutas, em K, da aleta e do ar ambiente. A convecção do calor é dada pela equação:

$$
q_{\text {conv }}=h \cdot A_{e} \cdot\left(T-T_{a m b}\right)
$$

Somando as equações (11) e (12) e rearranjando os termos obtem-se:

$$
q_{r a d}+q_{c o n v}=\left[h+\varepsilon \cdot \sigma \cdot\left(T^{2}+T_{a m b}^{2}\right) \cdot\left(\mathrm{T}+\mathrm{T}_{\mathrm{amb}}\right)\right] \cdot A_{e} \cdot\left(T-T_{a m b}\right),
$$

na qual os termos entre colchetes correspondem ao coeficiente de película "aparente", isto é, o valor experimental desse coeficiente obtido a partir do balanço térmico tradicionalmente empregado. Confrontando as equações (12) e (13), conclui-se que o coeficiente convectivo de transferência de calor, associado exclusivamente ao mecanismo de convecção, num ensaio real é obtido ao descontar do valor "aparente" a quantia dada por $\mathbf{h}_{\mathbf{r a d}}$, sendo esta última definida como:

$$
h_{\text {rad }}=\varepsilon \cdot \sigma \cdot A \cdot\left(T^{2}+T_{a m b}^{2}\right) \cdot\left(\mathrm{T}+\mathrm{T}_{\mathrm{amb}}\right)
$$

\section{METODOLOGIA}

No estudo proposto, foram utilizados os perfis de temperatura em duas barras cilíndricas horizontais, uma de alumínio e outra de cobre, com $1.150 \mathrm{~mm}$ de comprimento e $13 \mathrm{~mm}$ de diâmetro, aquecidas em uma das extremidades em um banho termostático e expostas ao ar ambiente. O equipamento utilizado está ilustrado nas Figuras 3 e 4 . Essas barras, que funcionam como aletas de seção circular, possuem orifícios para a introdução de termopares, que possibilitam registro da temperatura em vários pontos ao longo dessas aletas. A Tabela 1 indica a temperatura, em ${ }^{\circ} \mathrm{C}$, e a distância à base da aleta, em metros, em cada um desses 
termopares, após decorrido um tempo suficiente para a estabilização dessas temperaturas, caracterizando um regime estacionário. A temperatura ambiente, no ensaio, era de $20^{\circ} \mathrm{C}$ e as barras foram aquecidas a $85^{\circ} \mathrm{C}$ e $65^{\circ} \mathrm{C}$.

Tabela 1 - Registros de temperaturas ao longo das aletas

\begin{tabular}{|c|c|c|c|c|c|c|c|c|}
\hline & 0,05 & 0,10 & 0,15 & 0,25 & 0,35 & 0,45 & 0,60 & 0,75 \\
\hline $\mathrm{Cu}, 85^{\circ} \mathrm{C}$ & 59 & 56 & 53 & 41 & 34 & 29 & 28 & 24 \\
\hline $\mathrm{A} 1,85^{\circ} \mathrm{C}$ & 61 & 51 & 44 & 36 & 32 & 26 & 22 & 22 \\
\hline $\mathrm{Cu}, 65^{\circ} \mathrm{C}$ & 47 & 46 & 43 & 35 & 30 & 26 & 26 & 22 \\
\hline $\mathrm{A} 1,65^{\circ} \mathrm{C}$ & 49 & 41 & 36 & 31 & 29 & 24 & 22 & 20 \\
\hline
\end{tabular}

Figuras 3 e 4 - Equipamento utilizado nos ensaios, com destaque para o banho termostático (seta vermelha) e alguns dos termopares (setas amarelas).

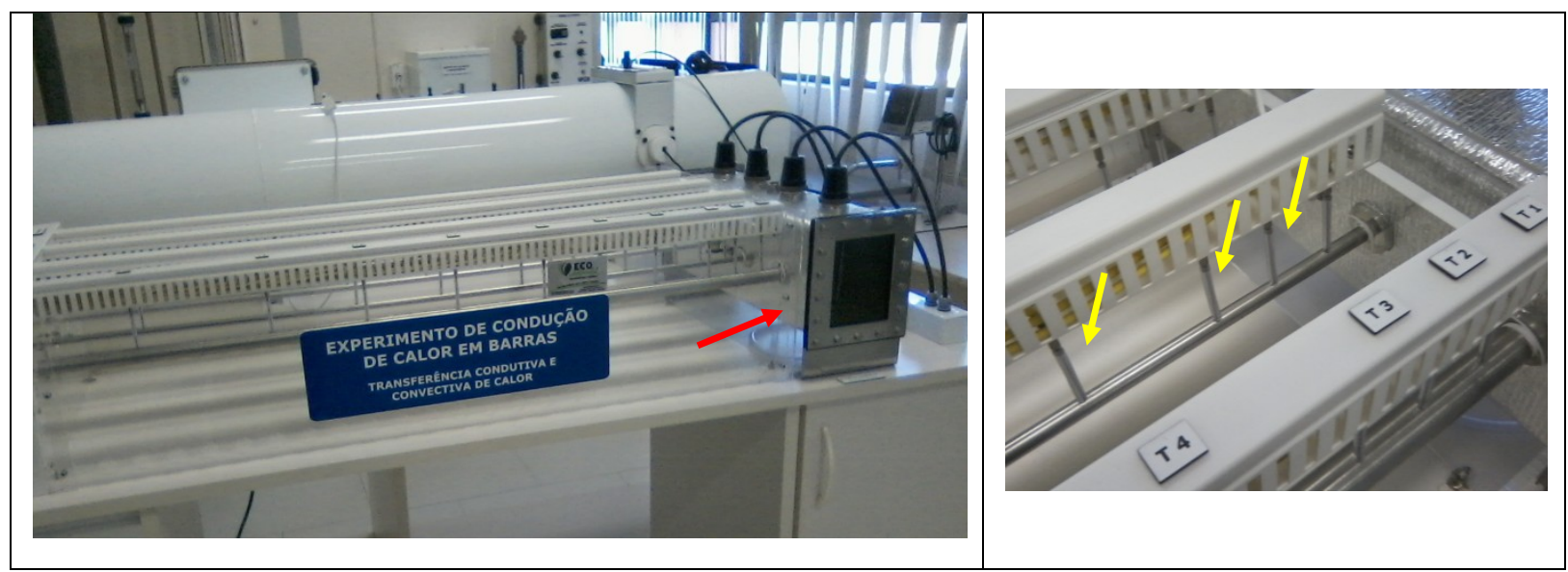

O ajuste para obter o valor de m é feito a partir da linearização da equação (4), que conduz a seguinte expressão:

$$
\theta_{*}=\ln \left(\frac{T-T_{0}}{T-T_{a m b}}\right)=-a \cdot x+b
$$

na qual $\mathrm{T}_{0}$ é a temperatura na base da aleta. A partir dos pares de valores $\boldsymbol{\theta} * \mathrm{e} \mathbf{x}$ associados a cada um dos quatro ensaios indicados na Tabela 1, obtém-se os coeficientes a e b da função indicada na Equação (15). O parâmetro m corresponde ao coeficiente a, e a partir dele determina-se o coeficiente de película "aparente", utilizando a Equação (2) e os valores da condutividade térmica do cobre e do alumínio (302 e $274 \mathrm{~W} /(\mathrm{m} . \mathrm{K})$, respectivamente).

$\mathrm{O}$ valor experimental de $\mathrm{h}$ associado ao modelo proposto resulta da diferença dentre o $\mathrm{h}$ "aparente" e o $h_{\text {rad, }}$, sendo este último calculado através da Equação (14). Nesta equação foi adotado um valor de $\varepsilon$ igual a 0,7 para as duas barras utilizadas, considerando a oxidação da superfície (KREIDER; KREITH, 1978). A Tabela 2 apresenta o valor do parâmetro m, em m

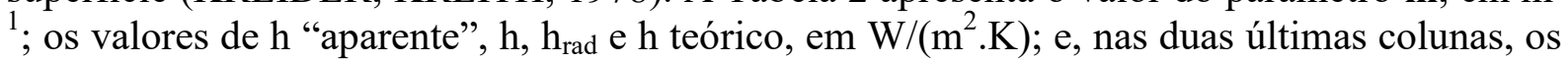
desvios relativos entre os valores de $\mathrm{h}$ "aparente" e h experimentais com o valor teórico desse coeficiente, identificados como I e II, respectivamente. O desvio relativo corresponde à diferença entre o dado experimental e o teórico, dividida pelo valor teórico e indicada em percentual. 
"Os desafios para formar hoje o engenheiro do amanhã"

Tabela 2 - Resultados obtidos nos cálculos realizados.

\begin{tabular}{|c|c|c|c|c|c|c|c|c|}
\hline & & $\mathrm{m}$ & $\begin{array}{c}\mathrm{h} \\
\text { aparente }\end{array}$ & $\mathrm{h}_{\text {rad }}$ & $\mathrm{h}$ & $\begin{array}{c}\mathrm{h} \\
\text { teórico }\end{array}$ & $\begin{array}{c}\text { desvio } \\
\text { relativo I }\end{array}$ & $\begin{array}{c}\text { desvio } \\
\text { relativo II }\end{array}$ \\
\hline $\mathrm{A}$ & $\mathrm{Cu}, 85^{\circ} \mathrm{C}$ & 3,29 & 13,09 & 4,71 & 8,38 & 8,85 & 47,87 & $-5,33$ \\
\hline $\mathrm{B}$ & $\mathrm{Al}, 85^{\circ} \mathrm{C}$ & 4,66 & 14,40 & 4,71 & 9,69 & 8,85 & 62,68 & 9,48 \\
\hline $\mathrm{C}$ & $\mathrm{Cu}, 65^{\circ} \mathrm{C}$ & 3,60 & 15,67 & 4,48 & 11,19 & 8,27 & 89,46 & 35,32 \\
\hline $\mathrm{D}$ & $\mathrm{A} 1,65^{\circ} \mathrm{C}$ & 4,68 & 14,52 & 4,48 & 10,04 & 8,27 & 75,59 & 21,45 \\
\hline
\end{tabular}

\section{DISCUSSÃO DOS RESULTADOS}

Analisando a Tabela 2, evidencia-se que os valores de $\mathrm{h}$ aparente são bem maiores que os valores correspondentes de $\mathrm{h}$ teórico, indicando que os desvios relativos entre eles não pode ser associado apenas a erros experimentais. $\mathrm{Na}$ comparação entre $\mathrm{h}$ e $\mathrm{h}$ teórico, os desvios relativos são significativamente menores; sendo que em um dos ensaios o valor teórico supera o dado experimental.

Diante do exposto, conclui-se que o balanço térmico tradicionalmente empregado na determinação do perfil de temperatura em uma aleta em regime estacionário, numa condição de convecção natural, resulta em valores de h que não condizem com as correlações empíricas utilizadas no cálculo desse coeficiente. A inclusão do mecanismo de radiação de calor no modelo tradicional, conforme proposto nesse artigo, produz valores experimentais de h que se aproximam mais daqueles previstos por essas correlações, simulando de forma mais adequada o processo de transferência de calor na superfície de uma aleta.

As correlações disponíveis para o cálculo de $\mathrm{h}$ a partir do número de Nusselt foram desenvolvidas, em sua maioria, até meados da década de 1970; e, segundo Boetcher (2014), eram focadas em métodos experimentais. $\mathrm{Na}$ convecção em torno de cilindros horizontais isotérmicos, o emprego dessas correlações pode produzir erros de até $20 \%$ na estimativa desse coeficiente (MORGAN, 1975).

No que diz respeito aos erros causados pelas condições experimentais, destacamos a variação instantânea de temperatura que ocorre ao longo da aleta; pois, mesmo após longo período de tempo, se verifica uma flutuação de até 2 graus em alguns termopares. Esta flutuação é produzida por correntes de ar repentinas, causadas, principalmente, pela movimentação de pessoas e abertura de portas e janelas; alterando o escoamento de ar induzido na superfície da aleta. Os erros de medição intrínsecos aos termopares, cuja resolução é de $1^{\circ} \mathrm{C}$, também contribuem para os desvios constatados nos ensaios.

Outro fator que se mostrou muito importante nos resultados foi a temperatura da base das aletas. No gráfico da Figura 5, são apresentados os desvios médios entre dados experimentais e valores teóricos nas duas temperaturas de base e nas duas metodologias de cálculo do valor de h. Os valores indicados apontam que, quando a temperatura da base é menor, os erros associados às condições experimentais tendem a aumentar. Isto decorre da menor diferença entre a temperatura da aleta e o ar ambiente; e, consequentemente na maior influência que as flutuações instantâneas de temperatura produzem no cálculo do parâmetro $\mathbf{m}$. Conforme visto na seção 3, são utilizadas as razões entre as diferenças, entre a temperatura da aleta e do ar, nos pontos onde estão instalados os termopares e na sua base, nesse cálculo. 
Figura 5 - Desvios relativos médios entre dados teóricos e experimentais de $\mathrm{h}$, confome o mecanismo de transferência de calor considerado e a temperatura na base da aleta.

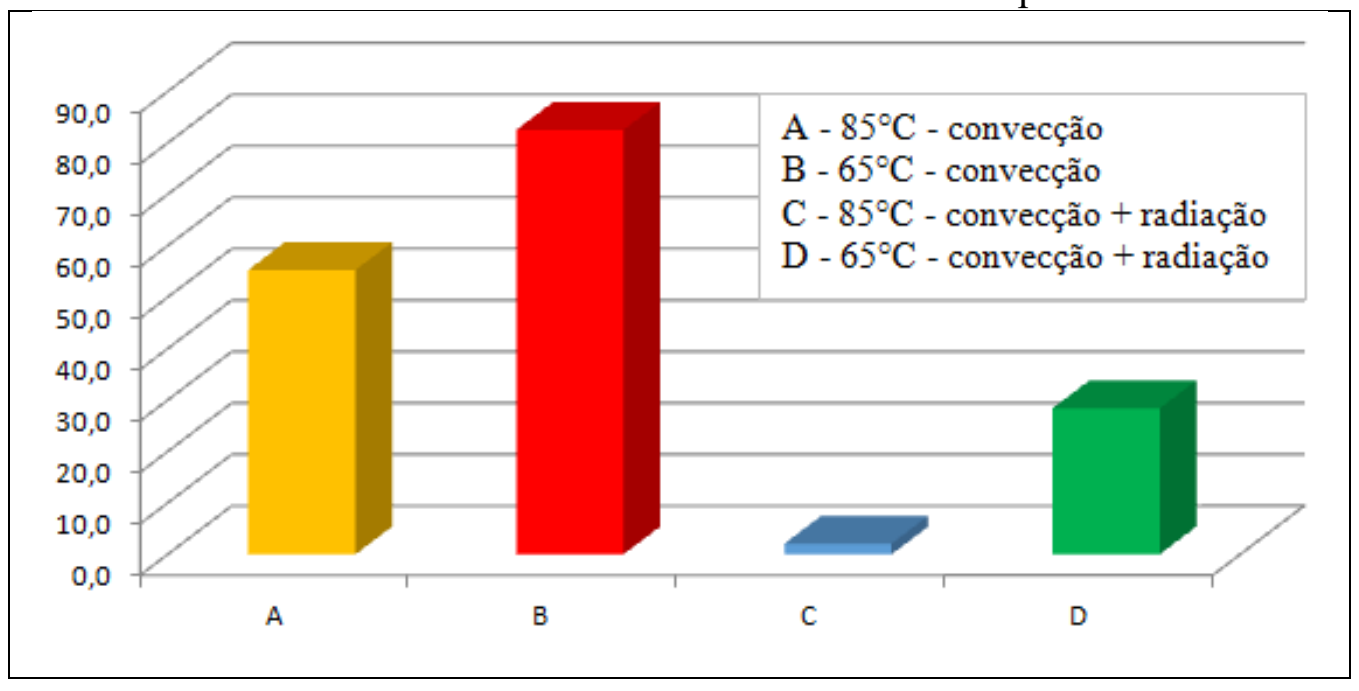

\section{CONCLUSÕES}

Os resultados quantitativos obtidos demonstram que a metodologia utilizada no cálculo de $\mathrm{h}$ para uma aleta, em regime estacionário e convecção natural, é válida. $\mathrm{O}$ estudo experimental da transferência de calor em uma aleta, segundo a proposta apresentada neste artigo, constitui uma alternativa que possibilita uma análise mais ampla do fenômeno da convecção do calor e, particularmente, dos valores de $\mathrm{h}$ utilizados nos problemas cuja resolução é solicitada em aulas expositivas convencionais. Paralelamente, no estudo proposto, o aluno é confrontado com a ideia de que radiação e convecção do calor ocorrem de forma simultânea; e, a abordagem tradicionalmente adotada, baseada no estudo em separado desses dois mecanismos, não pode ser aplicada em muitas situações reais.

Os ensaios realizados exigem o estudo de números adimensionais e o cálculo de alguns deles na investigação do comportamento de um sistema físico sob condições variadas. Essas tarefas levam o aluno a ter uma melhor compreensão da natureza e da importância desse conhecimento, bem como do emprego das correlações empíricas que envolvem os números adimensionais e o coeficiente convectivo de transferência de calor.

Os autores concluem que, embora muitas instituições de ensino não disponham de recursos financeiros para adquirir o tipo de equipamento utilizado no experimento descrito, é possível utilizar dados experimentais disponíveis na literatura para realizar o tipo de estudo proposto. Outra possibilidade é a construção de equipamentos com materiais de baixo custo; e, no caso do estudo da transferência de calor aletas, o banho termostático pode ser confeccionado a partir de uma caixa de isopor. As temperaturas ao longo das aletas podem ser registradas a partir de sensores de temperatura, do tipo PT 100, ou até mesmo com uma câmera termográfica, quando esta for disponível.

\section{REFERÊNCIAS}

ARAÚJO, Mauro S. T; ABIB, Maria Lúcia V. S. Atividades Experimentais no Ensino de Física: diferentes enfoques, diferentes finalidades. Revista Brasileira de Ensino de Física, v.25, n.2, p.176-194, 2003. 
BOETCHER, Sandra K. S. Natural Convection from Circular Cylinders. Ed. Springer, 48p, 2014.

ÇENGEL, Yunus A.; GHAJAR, Afshin J. Transferência de Calor e Massa: Uma abordagem prática. Editora Mc-Graw Hill, Porto Alegre, 2012.

GARCIA, Renato L. et al. Tanque hidráulico vertical para cálculo de perda de carga em tubulações. Revista Eletrônica Cientifica da UERGS, vol. 2, n. 1, p 54-62, Porto Alegre, 2016.

GIORDAN, Marcelo. O papel da experimentação no ensino de ciências. Revista Química Nova na Escola, v. 10, n. 10, p. 43-49, 1999.

INCROPERA, Frank P.; DE WITT, David. Fundamentos de Transferência de Calor e Massa. Editora LTC, Rio de Janeiro, 2008.

KREIDER, J. F.; KREITH, F., Principles of Solar Energy Handbook. Ed. Hemisphere Publishing Co., New York, 1978.

LIENHARD, John, H. A Heat Transfer Handbook. $3^{\text {rd }}$ ed., 2004.

MORGAN, Vincent, T. The Overall Convective Heat Transfer from Smooth Circular Cylinders, Advanced Heat Transfer, Vol. 11, pp. 199-264. 1975.

\title{
A TEACHING PROPOSAL IN FINS HEAT TRANSFER ON STEADY- STATE CONDITIONS IN QUIESCENT AIR
}

\begin{abstract}
In this work a didactic experiment about heat dissipation in circular fins, on steady state conditions, in quiescent air, proposing a modified method in order to quantify the convective heat transfer coefficient is presented. The results are compared with theoretical values predicted in empirical correlations, based on dimensionless numbers, for this coefficient, proving the fitness of the formulated proposition. The teaching strategy used in the formulation of this proposal and in the analysis of the results is compared with the traditional teaching model, highlighting the advantages of a practical class in order to minimize difficulties faced by students in learning heat transfer in fins.
\end{abstract}

Keywords: Fins. Heat transfer. Natural convection. Heat radiation. 